\title{
Rearing and Handling Children with Disability in Gumuz Culture: The Case of Mandura Woreda, Ethiopia
}

\author{
Zeleke Berie Gizachew Yihunie Daniel Seif \\ Gilgel Beles College Teachers Education
}

\begin{abstract}
This paper has the objective to assess the indigenous cultural practices of child with disability rearing and handling practices of Gumuz society. The research was conducted in one of the traditional societies in which indigenous child rearing is highly observed that is the Gumuz community in Benishangul Gumuz Regional State of Mundura Woreda. Both primary and secondary data were employed in this study. Qualitative method of semi-structured, participant observation, informal discussion and focused group discussions were utilized for primary data gathering. More over literature reviews, books, magazines and related researches were also used as secondary sources of data for the study. Eight kebeles' from the Woreda were selected purposively and a total of 85 respondents participated in this study. Men and women farmers who have a child with disability, community elders, and Gumuz teachers were included. Findings of the study show that there are various cultural practices in Gumuz community of child with disability rearing and handling practice, discrimination of children with disabilities and their parents, segregation of children with disability during child playing, domestic violence (pinching, beating, and physical punishment) are the major cultural practices that put children with disabilities at a lower status in Gumuz community. Furthermore, lack of awareness about the importance of education for children with disabilities is also another factor that puts children with disabilities in a lesser rank.
\end{abstract}

Keywords/phrases: Disability, Child rearing, Child handling

DOI: $10.7176 / \mathrm{JCSD} / 58-03$

Publication date: April $30^{\text {th }} 2020$

\section{Introduction}

The holistic development of children (i.e. physical, intellectual, social/behavioral and emotional development) cannot be seen independently of cultural practices and child rearing practices. Cultural practices are way of life that affects all aspects of human development by applying the knowledge of social and cultural factors that affect life's activities across multiple contexts (Linda, 2005).These life activities include children's emotional and social activities. Children's emotional and social development involves their thought and feelings about themselves and their relationships with others (Cobb, 2001).

Child development includes the physical, mental, behavioral, emotional and spiritual components of the child and they are incorporated in all child rearing, handling and cultural practices. According to (Cobb, 2001), child rearing practice includes maternal knowledge, beliefs, values, and parental styles that are influenced in a society. Since child rearing is viewed as a shared responsibilities(mother and father or extended family),a child's accomplishments reflect on the whole community(Este \& Sethi,2005).Cultural practices and childrearing practice are synchronized as the two sides of the same coin.

Child rearing plays crucial role in the development of children and is mainly carried out by family, which is the most sustained source of social contact for the child. The beliefs, values, and attitudes of the culture are filtered through the parents and presented to the child in a highly personalized, selective fashion (Hetherington \& perke, 1986).In addition, Ezewu (1998) noted that the type and quality of child rearing practiced by parents is critical because it allows children to learn and view themselves positively as competent, effective individuals able to influence events and people in the world around them. To achieve such competency Ezewu further noted that parents must permit children to take the initiative and assume responsibilities appropriate to their age-children need the support and freedom to apply, test, and practice social rules and skills, and they must learn by doing (Ezewu,1998).

While a task of children rearing is a great responsibility for parents, the challenges even lies when they have children with disabilities. Because of the nature of the problem disability brings in to the family, parents and other members of the family tend to react in different ways.

In a base line survey conducted in Ethiopia, Tirusew (1995) state that the experience of parents with children with disabilities is different. Their reaction when they know that their child is disabled varies; they may experience disappointment, anger, or guilt. He further indicates that these reactions are likely to occur soon after the discovery of the problem. Disappointment arises from awareness that the child will not be able to fulfill all fond hopes and expectations that have been built up even prior to his birth. Anger is initially directed at the child as the obvious sources of frustration of those expectations. In addition, guilt feelings arise from the need of parents to deny that they are angry at the child. One way of parents may deny this is by turning the anger inward at them. In the most extreme form, it has been found that anger toward the child for failing to fulfill ambitions can generate the desire 
to do away with the child (Tirusew, 1995).

In light of the above notion, addressing the issue of childrearing of children with disabilities at cultural level would contribute to the promotion of knowledge on prevailing rearing practices concerning children with disabilities, its implication on their wellbeing, and possible intervention mechanisms. It became evident that a lot of research has been done internationally, but not much locally. It is, therefore, professionally and ethically justifiable to look at as how children with disabilities reared and handled, as it could help us to understand the practices and early intervention programs in existence and to scholarly suggest possible options. That is why the researcher wanted to know how children with disabilities are reared and handled in Gumuz culture: The case of Mandura woreda, Ethiopia in a way that they can be independent and competent citizen of the society.

The general objective of this study was to investigate indigenous rearing and handling practices of children with disabilities in Gumuz society in the case of Mandura woreda, Ethiopia in order to identify factors that affect the general development in this community child with disability. Thus, the specific objectives of the study were to identify the types of the indigenous handling and rearing practice of children with disabilities in Gumuz people, to explore safe and harm rearing and handling experiences of children with disabilities in Gumuz people, to explore challenges in handling and rearing practices of children in Gumuz societies to grow and develop and to identify the major undesirable child rearing and handling practices in Gumuz societies.

\section{RESEARCH METHOD AND DESIGN}

\subsection{Research Design}

To achieve the objectives of the study the researchers used qualitative research methods. Such methods are helpful in obtaining a closer investigation and it is appropriate in order to listen to what people have to say about their real lives: as a matter of fact the potential for listening is a major advantage of the qualitative method over the quantitative one. In relation to this the researchers used (particular custom and characteristics (ethnographic) design of research which investigates the culture of the group. The researchers chose Mundura Woreda as research site because of the researchers' familiarity with the area and because the researchers had the opportunity to observe their traditional society in which children reared and handled. In addition, the Gumuz people live in a peripheral area of the country where they receive little attention from researchers.

\section{2 .Sources of Data}

This study employed both primary and secondary data sources. For this study, the primary data sources were the researchers as participant observation, men, women and Gumuz teachers from the community who are indigenous assumed to be key informants from eight selected kebeles of Mandura woreda. They were selected by their roles and responsibilities in these kebeles' assuming to have better information on the problem studied.

In addition to, the primary data, secondary data were employed. In this case, literature reviews, books, magazines and related researches were also used as secondary sources of data for the study.

\subsection{Sampling Techniques}

By using non-judgmental/purposive sampling, eight kebeles were selected. The total respondents who participated in the study were 85 selected purposely in close consultation with community leaders. The reason was they were assumed that they can give relevant data. The women, men and Gumuz teachers' respondents were represented to express their cultural issues in their kebeles'. More number of father and mother with child with disability respondents are included since the children with disabilities are the focus of the study.

Sixteen (16) women farmers and Twelve (12) men farmers were involved in the semi-structured interview. At least Equal numbers of both sexes are included since both respondents are the focus of the study. Particular respondents were systematically selected to illustrate cases. Twenty two (22) men community elders, Twelve (12) men Gumuz teachers, and twenty three (23) women farmers were selected based on their position and status in the community and involved in the focus group discussion. A purposive sampling technique was also used to determine to select good informants. This helped the researchers to acquire a variety of responses which is useful in reaching the general and specific objectives of the study.

\subsection{Data Gathering Instruments and Procedures for Data Collection \\ 2.4.1. Observation}

Participant observation was conducted to have an insight on the rearing and handling practices of children with disabilities in particular in the study area. The researchers participated in the routine life and different practices of people. Fortunately the researchers were already familiar with the area in different activities (in teaching in primary schools, High school, college, \&Facilitating the teaching learning process in woreda level).And it was not so difficult to involve in the culture, daily activities and the context. Participating in social activities was considered very important in order to create closeness and familiarity. The researchers as participants observed and collected field notes on the actual activities of the people. These include: cares of the child with disability, engagement of 
children with disabilities when they play together with others, accessibility of schools for children with disabilities and their way of participating in education, and in different cultural and traditional ceremonies. This approach helped the researchers to engage in more open informal discussions and obtain information about the attitudes, beliefs, and thoughts of the society in rearing and handling of children with disabilities.

\subsubsection{Semi-Structured Interview}

In this interview schedule, semi structured questionnaire items were developed to the key informants. The semi structured interview items have the advantages of flexibility in which new questions could be forwarded during the interview schedule based on the response of the interviews and enable to the researchers to ask for further clarifications of the issues under considerations. In this case, the process of interview was conducted in both Amharic and Sa-gumuza. The interview schedule were supported by Audio (tape recorder) in order to minimize loses of audio information. The recorded data were categorized based on similarities of responses and then transcribed in to English language to prepare for data analysis.

\subsubsection{Focus Group Discussion}

sixteen focus group discussions were also held with twenty two men community elders, twelve teachers and twenty three women separately (ten mothers, six grandmothers, one teacher of disability and two fathers of a child with disability are included), in order to express their feelings and opinion about the causes, prevention and remedy of disability in the community with confidentiality. These groups were approached because they have a significant role to play in the cultural and traditional practices of child with disability rearing and handling, plus this approach helped to compare the information with the data obtained through semi structured interviews. The results are useful to triangulate with in semi- structured interview, and observation. It was also to ensure that participants have something to say on the problems of the study within a short period of time.

\subsection{Procedures for Data Collection}

Before administering the instrument prior consent and willingness of the participants were asked orally. The semistructured interviews were asked in their home. Then after, the focus group discussions were also held with the participants in nearby home school compound. From these sessions, it aimed to obtain relevant data regarding the participants' personal perceptions and meanings, opinions, values, beliefs and feelings concerning the contents of children with disability in particular rearing and handling practice of their culture. By following these procedures, the researchers ensured triangulation of using multiple instruments, method of data collection and analysis. This has also enabled the researchers to test the validity of the study instruments.

\subsection{Data Analysis Techniques}

In organizing the primary data, the researchers listen and read again and again the recorded and the written information respectively obtained from observational notes, Semi-structured interviews, focus group discussion and documents that are helpful for the study were structured, organized and framed to suit analysis and interpretation.

In analyzing the data information were grouped into themes based on the general and specific objectives used in the study. Descriptive method was used that is narrative explanation, illustration of cases and discussions of the findings. Qualitative data analysis, the researchers kept records of what happened in the field in detail on every setting starting the first day in the field and then wrote the summary of every day notes. Finally, based on the findings and conclusions reached, recommendations have been proposed as a research output.

\subsection{Validity}

In order to control the validity of the data in this study, we used two techniques. First, in order to insure the proper understanding of the information sought, we briefly summarized the data and shared it with the research respondents and received feedback so that our further interpretation would be credible and trustworthy. In doing so, we completed a post data collection visit to parents and crosschecked the truthfulness of the data by reading the interview transcripts and making a brief discussion to cross check some of the data from the observation. Additional inputs that were obtained during the post data observation from the respondents in the form of feedback were also included in the data. Second, as argued by Wei and Moyer (2008), triangulation of the data from various sources (participants' semi-structured interview, focus group discussions, and observation) had increased the internal validity of the data obtained. Hence, the gap in one of these data sources can be strengthened and crosschecked by the other

\section{Findings, Interpretation and Discussion 3.1.Formal Schooling Practices}

Formal schooling is one aspect in the participants' rearing practice. In this respect half of the participants said they do not send their children with disabilities to school. While only two mother of children with disabilities send to school. Among those who reported that they do not send their children to school are children with disabilities that 
have mobility problem. A few participants were afraid of that the children with disabilities fail to get hurt, others said if insults with by other students.

In addition to the above reasons given by participants, in the discussion made with women groups, some information were obtained pertaining to the reason why children with disabilities are not going to school. Some of the dialogue includes the following:

"Majority families in our neighborhood who have children with disabilities do not send to school. Because parents want to keeps their name. They are afraid that people would know that they have 'Asheda ' or' basachugua'( Derogatory word for physical disability in Gumuz language and after words would point their fingers at them saying' Duwa Asheda' which means parents of children with disabled child".

Another mother participant of the group continued:

"For example, it is common to see mothers and fathers keeping out their children with disabilities from registered in 'universal primary education form'. We hear them saying 'we do not have children' when the teacher in nearby home school asks them to register their children. The reason why do this is because they think the child with disability does not have any use and educational right any way".

Concerning the educational progress of children with disabilities;

An important aspect of family activity that can have an impact on the educational progress of their children with disabilities is the kind and amount of support they get from parents. Pertaining to this point most of the participants in the semi -structured and focus group discussion said that: "Even if support is good, we do not know the kind of support is given whether material, verbal, and non-verbal encouragement".

In general concerning formal schooling, almost all participants do not send school their children with disabilities nearby school. Because of the schools are not accessible to children with disabilities .But, some of them said that they could give priority for the education of children without disability.

\subsection{Disciplinary Practice}

All participants in the focus group discussion reported that children with disabilities have behavioral problems, which include the following: "cries constantly, disobeys parents, talks to self like Satan, nervous, non-responsive, language- less, day dreamers, and aggressive".

Information from the parents in the semi-structured interview and focus group discussion further indicated that in the eyes of these parents children with disabilities are generally misbehaving than from children without disabilities .And the behavior of their children with disabilities are related to their disability.

Participants also asked to indicate the type of disciplinary actions they take on their children with disabilities:

"All women participants in the semi structured interview and focus group discussions said

that they use non-corporal punishment which includes advising and charming, but male

Participants use corporal punishment to correct the miss- behavior of the child whether with disability or not."

In general participants have found that mild corporal and non-corporal punishment to be the most effective way of disciplining children with disabilities.

Regarding disciplinarians all participants in the semi structured and focus group discussion agreed mothers should administer the disciplinary actions on their children with disabilities. The reason that they were given was that to help their child with disability have good future life, and to help children with disabilities harmoniously with family. A typical example here is the explanation of a mother of a child with disability in the semi-structured interview who supports these reason: "....I could punish my child until he knows his 'soul'....but once he become a young child he could control himself and do things as he is told...then I do not have to beat him..."

Another participant in the focus group discussion said that:

"It is difficult to apply corporal punishment on children with disabilities because it would make more disabled and unhappy. And children with disabilities is already cursed by 'Musa' (similar word for house sprite God), does not need additional punishment, disability is punishment by itself, so additional punishment is not necessary, because it can add another disability".

\subsection{Informal Gender-Role Skill Training Practice}

Concerning the expectations of participants with regard to skills that their children with disabilities should develop, most of mothers in the semi structured interviews said that:

"These children should try to perform domestic female skills like fetching water, collecting wood, cooking, and any other female works as their ability. A few were not agreed on the reasons that they gave children with disabilities need to use special instructions due to the disability and the inability to perform equally with the non-disabled, and the fact that children with disabilities need more time than the non-disabled to do or to finish a specific task. 
The participants practice also seems to be influenced by their excessive sympathy for their children with disabilities, and possibly due to their underestimation of children with disabilities.

As one mother said about her child with mild intellectual disability:

"My daughter deserves to spend her time by playing only. She wouldn't have to suffer more than what she does already. To make her do things in the house wouldn't be cruel of me. I wouldn't allow that to happen to her as long as I am alive..."

The last reason is a grandmother of twelve- year- old cerebral palsy girl (hemiplegic) said:

“...she sometimes fetches water, but it takes long time... with the other siblings I couldn't have patience, as she has 'Basachugua' (derogatory word for physical impairment in her language) and I couldn't expect her to do and finish the task quickly..."

Regarding their male children with disabilities all participants in the semi-structured interview and focus group discussions said that "they must do things with in their capacity if possible".

Overall, the study indicates that parental practice with regard to providing informal gender role skill is biased due to their belief and their regard for children with disability. This is critical issue because daily living skills are fundamental to the personal and social development of children with disability (Hewared \&Orlansky, 1988).

The results concerning informal skill training show that there is no indication that participants' over burden the children with disability with work. However, the fact that almost all participants said that they do not provide informal gender role skill training to their children with disability due to their disability, could be considered as an element of maltreatment because that denies the children with disabilities the development of their knowledge and skills on activities useful for daily living. Such behavior or practice of parents could be the result of the belief and low regard they have towards the ability of children with disabilities. It is also attributed to the lack of time or special skill/know how on the parents side to train their children with disabilities.

\subsection{Beliefs of Parents on Causes, Prevention, and Remedies of Disabilities}

All participants in the semi- structured interview and focus-group discussions said that disability is not preventable because it is the result of house sprite 'Musa', oaths, when the mother of the child with disability had sexual meeting with the relative of her husband, when the mother got birth from her relative and man is unable to control its happening. The participants' response could be explained from the point of their traditional beliefs. This findings has consistency with the findings of Tirussew (1995) in a base line survey on disabilities in Ethiopia has also found that more half of the participants in the survey ascribe the cause of disability to be spiritual sources.

Concerning remedy of disability, all participants in semi-structured interview and focus group discussion said that they believe that their children with disabilities could be improved or cured through 'Musa' permits it. And also the treatment adopted by many mothers' also relates to make sacrifices for evil spirits to cast them out of their children's body.

"In this regard, a mother who found that her son has Epilepsy said," As soon as I understand my child has 'mental problem', I knew that my child had the 'likift';(the devil sprite) so I immediately consulted a very good 'gafiya' (similar word for awaki in Gumuz language), who told me that I should act fast before the likift controls my child's mind. So, I have done all my best following the orders of this 'Gafiya'...I have slaughtered three hens with different color by the house of me, and kept the blood on the floor for three days... but my son did not show any improvement, so I will take to him to another known 'gafiya'.

"Another grandmother of a child with multiple disabilities said," ones upon a time my neighbor's child has died. Because of this, the child's parent shouted at me, they consider me as an evil sprite that ate his child. The elders intervene to create an agreement by giving my daughter's for the parents of the died child through conducting an oaths (the oaths were, if a child dies due to my evil sprite, the child my daughter would bear be without disabled, if not disabled). After few years my daughter gives first birth children disabled and the second also, the second died after three years. Finally they send my daughter and her child with disability to me. Still I am living with the son of my daughter with disability.

The elaboration given above explains that the mother's belief on prevention and cause of disability influences their methods of intervention. Early identification is said to be the major and important aspect of disability intervention. According to Tirusew (1996) age 0-3 years is the ideal and critical age for early intervention of disabilities. However, though the mothers have identified their children's disabilities with in this period of time, it seems that they did not attempt to follow effective means of early intervention mechanisms.

Aside from religious orientations, traditional beliefs and low level of education, one reason why parents sought spiritual solution more than scientific ones could be attributed to lack of information on the parents' side on the services availability in their living area.

The above results neither show that the belief of the parents on the causes, prevention and remedy of disability is generally nor related to the scientific explanations. Scientific explanation of the causes of disability includes chromosomal abnormality, metabolic disorders, radiation, high consumption of alcohol by mothers, some antibiotics, post natal handicapping conditions, nutritional effects, and child abuse(Tirusew, 1996).Regarding 
remedy of intervention, a wider service is suggested by David and Orlansky(1988), and these include independency, including mastery of daily living skills, self-awareness and social maturation, academic growth, and career education, including constructive leisure activities. David and Orlansky (1988) also suggested that the intervention effort must involve interdisciplinary team of professionals and parents.

\subsection{Parent -Child Relationship}

The quality of relationship between parents and their children plays a determining role with the emotional and cognitive development of children in general, and on children with disabilities in particular (Rye, 1997).Spending more time with and talking to children enriches child-parent relationship.

The results of the study show that parents of the study have negative and deny relationship with their children with disability. The participants also said that the more caring person with whom children with disability is the mother and grandmother.

But, few participants in the interview said that: "Children with disabilities do not like to talk to other siblings or parents because they are always aggressive or always complain and/or not listen to others they feel inferiority. So, they are not interested to integrate with other parents and communities."

Play is another important means of physical, emotional, social, and intellectual development. This particular point is specifically valid during the early years of the child's life in the home.

\subsection{Understanding Parents Knowledge's about the Rights of Children with Disability}

To understand parents' knowledge about rights of children with disabilities, most participants in the semistructured interview and focus-group discussion were aware of the rights of a child with disability, but they indicated that children's right were not supported in the community. They felt that society did not display positive attitudes towards families that had children with disability and children with disabilities. These children should be given love and care and training as well. Concerning love and care one mother of a child of with epileptic said: "My son understands others' behavior and when somebody says anything wrong or scolds him he reacts and shows his aggression. We should treat them with love and care".

Only few Mothers in the focus-group discussion identified "formal education" as an important right of children with disabilities. They perceived it as a way of socialization for their children, and a stepping stone towards an inclusive community. But, almost all participants in the semi-structured interview said that:

"The mother is supposed to take good care of their health and cleanliness, People in our area is not good with children with disabilities, they make fun of them, even they dislike observing them and for the reason we do not let our children go outside the home, we hide them from protecting death".

From the above sayings we can easily understand that, the negative attitude of the community could be due to cultural and superficial beliefs connected with disability. Consequently, the families could not expect support or help from others, and were isolated from society. The burdens of care giving, with or without formal or informal support, often clearly overstretched the families' resources and affected each member in the household but particularly the primary caregivers. Therefore, the care of a child with disabilities not only depends upon the mother but also relies on all to provide the required education, training and support services.

In general, the data gathered showed that most of the participants identified the following needs: material needs (food, shelter, playing goods (toys)); influence social needs (parents, family, and friends); educational needs; psychological needs (need to be recognized, moral support, love, and care) as the most important needs of children with disability aged from $0-18$ years.

While most of the caregivers were aware of the children's basic universal needs, it is evident that some of these needs were minimally met, while others were not met at all. As Zimba (2004) Children in some families had their survival needs met, including access to food and shelter and protection from harm, to a certain extent. Arguably, the observation made revealed that a lot of dangerous objects, such as knifes, hammer and javelin were the playing objects of the Gumuz children and these objects may harm them. And also the negative attitude of the community initiates children with disabilities to become hiding in the house.

According to the UNICEF Report (2010) on promoting the Rights of Children with Disabilities, both children with disabilities and their parents face number of difficulties that stop them enjoying their basic human rights. "Their abilities are overlooked, their capacities are underestimated and their needs are given low priority" (UNICEF, 2010, p. 7).

Moreover, needs associated with psychosocial and other areas of well-being were not met at all for the majority of Gumuz children. Children's developmental niches are created not by their parents, of course, but also by aspects of the wider culture, such as, an urban or rural environment or unfortunately by major social dislocations, including poverty and alcohol abuse (Harkness \& Super 2008).

It was observed that many children with disabilities in these communities lived with mothers in overcrowded houses, and such children were depended on mothers. This led to the conclusion that child-care and support in these communities was left to mothers and grandmothers, who are usually involved in both food production and 
income generation.

Based on the observation made by the researchers, let alone Gumuz children with disabilities many Gumuz children without disabilities wore unclean, torn, oversized cloths, few children bellow aged ten seen without cloth and others looked neglected. The researchers reached the conclusion that poverty was some of the problems which hindered child with disability survival and development among the Gumuz children with disabilities.

Filling gaps in meeting the Gumuz children's with disabilities needs has become more significant. Even those who participated in this study have become aware of this gap and urged that the needs of these children can only be met with the involvement of 'everyone', that is, the whole family, including fathers, the community, community-based organizations, faith-based organizations, civil societies and government. These findings lend support to the ecological system theory which holds that the developmental process does not occur in a psychological vacuum but rather that individual child development is influenced by factors in the immediate environment, as well as society and culture as a whole (Track wick-Smith, 2007). From the data gathered from this study, it appeared that these caregivers seem to urgently need resources for child-rearing and not only mere knowledge and skills about how child-rearing should be done.

\subsection{Understanding Parents Knowledge's about how they Promote Intellectual and Language Development of their Children With Disability Through Socialization}

In addition, to understand how Gumuz parents reared and handled in promoting intellectual development among their children with disabilities by the process of socialization, one of the questions enquired about activities which children engaged in when playing with others.

Some of the activities mentioned require the use of large muscles in the legs or arms, as well as general strength and stamina (gross motor abilities), while others coordinate smaller muscles in the arms, hands and fingers (fine motor abilities).As female participants in the focus-group discussion discusses:

Examples of activities which enhance gross motor development are:

"Boys like to play with locally produced balls, girls imitating pounding millet, collect sticks and build toy houses and kraal ( a traditional African village of huts, typically enclosed by a fence), play hide and seek, Play pretending to protect their properties, while singing "I eat from there, you won't", boys like fighting, children like athletic/chasing each other, climbing trees, boys spike (javelin) throwing"

Activities which enhance fine motor development are:

"They can play 'children' with different fruits or with toys which is made of soil, Use old cloths to dress their fruits "children" ,Build toy houses with sticks, collect sticks and build toy houses, children like athletic/chasing each other".

The Semi structured question which enquired during focus group discussions about how children with disabilities could develop their language .All participants rise different mechanisms such as:

Most of the activities mentioned by the participants help children in acquiring new words/pronouncing words. The following statements were examples of the participants saying:

"poems- the child increase vocabulary, song-the child learn new words ,Ask a child to give object's names - practice words, telling each other story - learn to pronounce words, sing traditional song- a child practice words ,"repeat after me" - a child catch up new words, imitating parents- a child learn words one-byone , and also by describing features of objects."

The data gathered on the use of socialization to stimulate development revealed that all the participants sought:

"to raise children who were honest, obedient and responsible people (who were not expected to steal, lie, use bad language, fight, smoke, abuse alcohol and treat others with disrespect).

These findings support the findings of Zimba and Otaala (1995) who found that parents aimed at shaping children into honest, obedient and responsible persons. Gumuz goals of child-care which include cultural scripts such as developing obedience to authority and respect for senior members of the community resembles the macro system that Bronfenbrenner talks about. In this regard, the researchers observed that Gumuz children were not greeting adults while standing, but this action was observed with Gumuz children. The message that could be communicated is that, greeting adults while sitting instills listening skills and it also helps caregivers to observe the emotional state of the children. In addition, the belief system employed by Gumuz caregivers/parents is different from the modern way of raising children, as stipulated in the parental ethno theories subsystem of the development niche: that parents everywhere use their ethno theories to bring up their children to be happy, healthy, well-functioning and successful in their own culture (Myers, 1992).

Furthermore, activities such as building houses with sticks by boys and imitating pounding Millet (maize) by girls were important parts of psychosocial skills. These activities enabled children to become competent members of their communities 


\section{Conclusion and Recommendation \\ 4.1.Conclusion}

The results of the study shows that the parents rearing of their children with disability is positive but affected by their belief, sympathy, and low regard towards disability, and their socio-economic limitations such as lack of adequate income and education, and large family size.

With regard to their belief, the fact that the majority of parents believe that disability is caused by supernatural forces has negatively affected parental rearing specially in finding solutions to parenting problems of their children with disabilities. Parental low regard and sympathy towards their children with disabilities also affect negatively the schooling and informal skills training provisions to children with disabilities. With regard to disciplining their children with disabilities, parents has shown a favorable practice but with tendency of using more corporal punishment on child in future. These incidences, in general, show element of maltreatment.

In case of discrepancies on beliefs related to rearing practices, parents have greater negative beliefs towards their children with disabilities than their non-disabled children.

Aside from beliefs, in general, parental rearing of their children with disabilities is found to be positive .Despite their socio-economic constraints, some of the parents have provided their children with disabilities with basic requirements.

When it comes to intervention, the parents have shown low emphasis in putting the basic knowledge about the "scientific" characteristics of disability. Rather their concerns are on other but crucial intervention areas such as social, emotional and educational (formal and vocational) supports for the rehabilitation of children with disabilities. But interestingly, despite their economic limitations the parents did not emphasis economic input as means of rehabilitation for their children with disabilities.

In general, it is observed that a better perspective of the rearing practices by parents of the children with disabilities could have been achieved if a larger group of the children with disabilities, their family members such as the non-disabled siblings and the father of the children with disabilities were involved. This was not possible for lack of time and other engagements. Since the purpose of the study was to do an intensive investigation, it was deemed more appropriate to use reasonably low number of participants.

\subsection{Recommendations}

Culture and traditional practices play indispensable part in our lives and they have important effects on the way of life of individuals especially on the moral formation of a people. Individuals cannot depend on themselves alone to track their way through life. Hence those cultural beliefs, norms and customs that can hinder peoples from fulfilling equal rights in the society and prevented them from enjoying their economic, social and political rights should be examine.

Discrimination of children with disabilities existing in societies of every degree of complexity is so integral to society's culture. Addressing the cultural basis of children discrimination requires recognition of the means by which we are socialized to accept and perpetuate a reduction image of children with disabilities' achievements and capacities. In light of the findings, the researchers would like to suggest the following recommendations.

1. Since most parents in the study have negative belief about children with disabilities and lack the knowhow on how to help their children in rearing and handling for their development, they require professional help. So concerned family members, community, nongovernmental and governmental organization should extend their service to these children.

2. At family level children without disability play important role in educating the parents and family members around disability intervention. The siblings shave opportunities of contacts with other social systems outside the family specially the school. The school is sustainable institutional forum for the education programmed due to inaccessible information towards "Scientific" information regarding children with disabilities welfare. Education and awareness raising for the parents and their children with disabilities about "scientific" explanations on causes, prevention, and remedy of any disabilities, to change the present understanding of parents that is biased by their beliefs.

3. Those governmental and nongovernmental organizations that are working in Benishangul Gumuz, particularly on children in general and children with disabilities in particular and related issues needed to change the cultural stereotypes attached to children with disabilities as secondary and discrimination. This can be possible through continuous awareness creation program. There should child rights awareness within the community, among family members, \& schools. This could be done through advocacy in the family, community, \& in the school on the issue of convention on the right of the child with disability. Disability should be seen as quality of humans and the family, the community, and the society at large has to take one another's peculiarities in to account.

4. Benishangul Gumuz Educational Office needs to promote the importance of girls and children with disabilities education and teach parents to send their daughter and children with disabilities to school. Because education is one way in which those children can emancipate themselves from the grip of culture 
through personal domination. Finally it is recommended that comprehensive study that covers wide population in all areas must be taken.

\section{References}

Cobb N.J. (2001). The Child: infants and children. California. Mayfield published company.

Edwardraj S.et al, (2010). Perceptions about intellectual disability: a qualitative study from Vellore, South India. Journal of Intellectual Disability Research; 54 (8); pp. 736-748

Este,D and Sethi, . (2005).Factors influencing the child rearing practice of recently migrated Chinese Age 06.Journal of Adult Education Quarterly. Vol. 119.Retrieved on May 29/2008 form http://www,aeq.sagepub.com

Ezewu, E.(1998).Sociology of Education. Singapore: Addsion Wesely Longman.

Hetherington \& Parki (1986).Child psychology ( $3^{\text {rd }}$ ed.) New York: MCGraw-Hill Book Company.

Hewared,W.L. \& Orlansky,(1988) Exceptional children ( $3^{\text {rd }}$. ed.) Columbss: Merrill publishing company.

Linde,T.V. (2005).Influencing and Developing Good Policy in Early Childhood Development (ECD) Among Pastoralist Communities' in East Africa. The case's of Sambori in Kenya. Retrieve on August 28/2008 http:www.Berndvawleer,org.pvlp publication

Rye,H.(1997). A new international direction in initiatives for children with special needs. International consultation early childhood and special educational needs UNESCO, Paris

Tirussew Tefera (1995). Base line survey on disabilities in Ethiopia. Addis Ababa: Institute of Educational Research, Addis Ababa University.

Tirussew Tefera (1996). Persons with Disabilities of high achievement profile and resilience in Ethiopia. Addis Ababa: Radda Barnen (save the children Sweden).

Trawick -Smith, J. (2007). Early Childhood Development: A multicultural perspective. New York. Printing Hall. UNICEF. (2010). Defining quality in Education. New York. Miske Witt and Associates Publication.

Wei L., and Moyer M., G. (2008). The Blackwell guide to research methods in bilingualism and multilingualism. Victoria; Blackwell Ltd.

Zimba, R. F. \& Otaala, B. (1995). The family in transition: A study of child-rearing practice and belief among the Nama of the Karasa and Hardag regions of Namibia. Windhoek, Namibia: UNICEF

Zimba, R. F. \& Zimba, E. (2004).Review to determine whether current legislation on children in Namibia are in conformity with the convention the rights of the child. Windhoek, Namibia: UNICEF 